\title{
A design proposer on policy framework in IPV6 network
}

\begin{abstract}
Networked systems are increasingly driven by changing business needs, their management becomes even more complex. In order to adapt to changing business requirements, distributed systems switch from the traditional client-server model to a service-driven model: The internet service driven network is a new approach to the provision of network computing that concentrates on the services you want to provide. These services range from the low-level services that manage relationships between networked devices to the value-added services provided to the end-users. The requirements for management systems identified above, can be facilitated with policy-based management approach where the support for distribution, automation and dynamic adaptation of the behaviour of the managed system is achieved by using policies. IPV6 is one of the useful delivery protocols for future fixed and wireless/mobile network environment while multihoming is the tools for delivering such protocol to the end users. This paper proposed interface selection mechanism while the study presents a policy framework in IPv6 multihomed mobile network, policy Framework Architecture, and a case study for the framework.
\end{abstract}

Keyword: IPV6; Policy-based management; Multihoming; Policy framework 